\title{
Combining Probabilistic Shape-from-Shading and Statistical Facial Shape Models
}

\author{
Touqeer Ahmad ${ }^{1,2, \star}$, Richard C. Wilson ${ }^{1}$, William A.P. Smith ${ }^{1}$, \\ and Tom S.F. Haines ${ }^{3}$ \\ 1 Department of Computer Science, The University of York, UK \\ ${ }^{2}$ School of Science and Engineering, LUMS, Pakistan \\ 3 Queen Mary University of London, UK \\ \{touqeer,wilson, wsmith\}@cs.york.ac.uk, tom.haines@eecs.qmul.ac.uk
}

\begin{abstract}
Shape-from-shading is an interesting approach to the problem of finding the shape of a face because it only requires one image and no subject participation. However, SfS is not accurate enough to produce good shape models. Previously, SfS has been combined with shape models to produce realistic face reconstructions. In this work, we aim to improve the quality of such models by exploiting a probabilistic SfS model based on Fisher-Bingham 8-parameter distributions $\left(\mathrm{FB}_{8}\right)$. The benefits are two-fold; firstly we can correctly weight the contributions of the data and model where the surface normals are uncertain, and secondly we can locate areas of shadow and facial hair using inconsistencies between the data and model. We sample the $\mathrm{FB}_{8}$ distributions using a Gibbs sampling algorithm. These are then modelled as Gaussian distributions on the surface tangent plane defined by the model. The shape model provides a second Gaussian distribution describing the likely configurations of the model; these distributions are combined on the tangent plane of the directional sphere to give the most probable surface normal directions for all pixels. The Fisher criterion is used to locate inconsistencies between the two distributions and smoothing is used to deal with outliers originating in the shadowed and specular regions. A surface height model is then used to recover surface heights from surface normals. The combined approach shows improved results over the case when only surface normals from shape-from-shading are used.
\end{abstract}

\section{Introduction}

Shape-from-shading(SfS) is a method of finding the shape of a surface from a single image, and has been used previous for finding 3D face models. Faces are quite suitable for this approach because they have a fairly constant albedo over most of the surface (excluding eyes, facial hair and so on) and the shape varies smoothly. Capturing a face in this way only requires one image and no subject participation and so it is non-invasive and can be done at a distance. However,

\footnotetext{
* Touqeer Ahmad is thankful to EURECA scholarship scheme for fully funding his M.Sc. at University of York.
} 
even state-of-the-art SfS methods are not accurate enough to produce correct face shapes on their own.

In order to improve the quality of the face shape, a model is required. These may vary from specific constraints on the shape to complete models of the shape of faces; for example Prados and Faugeras [16] enforce convexity through the location of singular points. Zhao and Chellappa 17] use the symmetry of faces as a constraint. Samaras and Metaxas [18] use a deformable model of face-shape. Blanz et al. 6] exploit a full 3D morphable head model built from laser range scans which also includes texture. By minimising the difference between the rendered model and the measured face image, they can estimate face-shape, pose and lighting at the same time. Smith and Hancock 15 combined SfS with a statistical model of face shape. This face model was derived from 3D face range scans and provided sufficient constraints on the shape to produce realistic models of faces. This method exploited the SfS method of Worthington and Hancock [3] However, Haines and Wilson [1] presented a more reliable SfS algorithm which use directional statistics; specifically Bingham, Bingham-Mardia, von-Mises-Fisher and Fisher-Bingham 8-parameter distributions $\left(\mathrm{FB}_{8}\right)$ [10] to model different entities involved in any SfS algorithm Cone constraint [3], boundary and gradient information and resulting surface normals [1. In our work we make use of advantages of both of these models and combine the statistical face shape models of Smith and Hancock with probabilistic Shape-from-Shading method of Haines and Wilson in a probabilistic framework to give a more reliable facial Shape-from-Shading method.

These two models produce results on different spaces and using different probability distributions. The SfS algorithm of Haines and Wilson outputs the surface normal directions on the unit sphere as Fisher-Bingham distributions $\left(\mathrm{FB}_{8}\right)$ for every pixel whereas statistical model of Smith and Hancock gives a Normal distribution on the tangent plane for each pixel. The two models can only be combined when they are in the same space and are represented using tractable distributions.

\section{Background}

This research work aims to improve the quality of SfS face-shape models by combining a probabilistic Shape-from-Shading method with a statistical shape models of human faces. The goal is to exploit the distributions provided by the two methods to locate a maximum-likelihood shape which accounts for the uncertainties in both models. To commence, we briefly describe the formulation of the two models, and the exponential map, which form the key elements of our framework.

\subsection{Shape-from-Shading}

Haines and Wilson presented a probabilistic Shape-from-Shading algorithm based on Markov random fields and belief propagation [1. Directional statistics, specifically $\mathrm{FB}_{8}$ distributions [9] were used for the probabilistic representation of surface 
orientation. In particular, the method produces a $\mathrm{FB}_{8}$ distribution describing the surface normal at each point on the image

$$
p_{\mathrm{FB}}(\hat{\mathbf{x}} ; \mathbf{A}) \propto \exp \left(\mathbf{b}^{T} \hat{\mathbf{x}}+\hat{\mathbf{x}}^{T} \mathbf{A} \hat{\mathbf{x}}\right)
$$

where $\hat{\mathbf{x}}$ is the surface normal direction, $\mathbf{b}$ is the Fisher parameter and $\mathbf{A}$ is Bingham matrix and is symmetric. In this method, the irradiance and smoothness constraints are defined in terms of directional distributions. The global distribution of normals is described using a Markov random field model. Haines and Wilson used the hierarchical belief propagation method of Felzenszwalb and Huttenlocher 11] to find the marginals of the directional distributions; once the belief propagation has converged the Shape-from-Shading algorithm gives surface orientation for each pixel using an $\mathrm{FB}_{8}$ distribution. Haines and Wilson's approach generally performed well as compared to Lee \& Kuo[2] and Worthington \& Hancock [3] and has the advantage of characterizing the uncertainty in normal direction at any point.

\subsection{The Exponential Map}

Directional data can be naturally modelled as points on the surface of a unit sphere. The direction is given by the unit vector from the origin to the point on the surface. This is a non-linear manifold which highlights some of the difficulties in computing statistics with surface normals.

The exponential map is a map from points on the manifold to points on a tangent space of the manifold. As the tangent space is flat (i.e. Euclidean), we can calculate quantities on the tangent space in a straightforward way. The map has an origin, which defines the point at which we construct the tangent space of the manifold. Formally, the definition of these properties as follows: Let $T_{M}$ be the tangent space at some point $M$ on the manifold, $P$ be a point on the manifold and $X$ a point on the tangent space. We have

$$
\begin{gathered}
X=\log _{M} P \\
P=\operatorname{Exp}_{M} X
\end{gathered}
$$

The Log and Exp notation defines a log-map from the manifold to the tangent space and an exp-map from the tangent space to the manifold. This is a formal notation and does not imply the normal log and exp functions - although they do co-incide for some types of data, they are not the same for the spherical space. $M$ is the origin of the map and is mapped onto the origin of the tangent space.

For the spherical manifold, which is used to represent directional data, the log-map corresponds to the azimuthal equidistant projection, and the exp-map to its inverse. We define a point $P$ on the sphere as a unit vector $\mathbf{p}$. Similarly, the point $M$ is represented by the unit vector $\mathbf{m}$ which is the origin of the map. The maps are then

$$
\begin{aligned}
& \mathbf{x}=\frac{\theta}{\sin \theta}(\mathbf{p}-\mathbf{m} \cos \theta) \\
& \mathbf{p}=\mathbf{m} \cos \theta+\frac{\sin \theta}{\theta} \mathbf{x}
\end{aligned}
$$


where $\theta$ is the angle between the vectors. The vector $\mathbf{x}$ is the image of $P$ and lies in the tangent space of the sphere at point $\mathbf{m}$, and the image of $M$ is at the origin of the tangent space. $\mathbf{x}$ is intrinsically 2-dimensional as it lies in the tangent plane, and we can characterise it by two variables if we define an orthogonal coordinate system for the tangent plane using the unit vectors $\left\{\mathbf{u}_{1}, \mathbf{u}_{2}\right\}$; the two parameters are then $\alpha=\mathbf{x}^{T} \mathbf{u}_{1}$ and $\beta=\mathbf{x}^{T} \mathbf{u}_{2}$.

\subsection{Facial Shape Model}

Smith 4] constructed a statistical model of needle maps using range images from 3DFS database [5] and Max Plank database [6] based on Principal Geodesic Analysis [7. Each range image in the training data is converted to a field of surface normals and then PGA is applied to the data. Smith calculates the spherical median $\mu(x, y)$ at each pixel location. At each point in the image, a tangent plane to the sphere is defined by the direction $\mu(x, y)$ and the surface normals projected onto this tangent plane using the log-map. The covariance matrix calculated using PGA then describes the distribution of surface normals on the union of all of these tangent planes. As a result, the statistical model consisted of the set of spherical medians $\mu(x, y)$ and a covariance matrix on the union of tangent planes $\mathbf{L}$.

Smith also constructed a statistical surface height model which gives surface height from surface normals implicitly without following any explicit surface integration method. Like the statistical surface normal model Smith's height model extracts the principal modes of variation from a joint model of surface normal and height. Least-squares can then be used to locate the best height model for a particular configuration of surface normals.

\subsection{Outline of the Method}

Our goal is to combine these two probabilistic descriptions of the set of surface normals, one from SfS and one from the shape model. The $\mathrm{FB}_{8}$ distribution is difficult to manipulate; for example finding maximum-likelihood estimates for the parameters is a non-trivial problem. Instead, we convert the SfS distributions to distributions on the tangent planes of the shape model using sampling. We commence with the the $\mathrm{FB}_{8}$ distributions delivered by SfS and the Smith and Hancock statistical face model. The first step is to sample the $\mathrm{FB}_{8}$ distributions. From these samples we compute the spherical median at each point. We then project the samples onto the tangent plane of the directional sphere and compute the Normal distribution on the tangent plane. We then combine this with the Smith and Hancock model on the tangent plane to provide a combined distribution of surface normals. We identify and eliminate SfS outliers using the Fisher criterion. Finally we smooth and integrate the resulting surface normals to obtain a shape estimate.

In section 3, we describe a sampling method for the $\mathrm{FB}_{8}$ distribution. In section 4 we explain how to model the samples on the tangent plane and combine them with the shape model. Section 5 describes how to identify outliers where 
the SfS is ineffective, and how to construct a final face shape. Finally, we present some results in Section 6.

\section{Sampling of $\mathrm{FB}_{8}$ Distributions}

The $\mathrm{FB}_{8}$ distribution 9 is a multivariate normal distribution that is constrained to lie on the surface of a unit sphere. The Fisher-Bingham distribution is used to model the directional data on spheres and sometimes for shape analysis 1 . If $\mathbf{x}=\left(x_{0}, x_{1}, x_{2}\right)$ is a random variable from this distribution then according to the unit norm constraint $\|x\|^{2}=1$. Hence, $\left(x_{0}^{2}, x_{1}^{2}, x_{2}^{2}\right)$ lies on a simplex.

Our goal is to generate a set of samples from the distribution using the method of Kume and Walker 9] which we briefly describe here. The key idea of Kume and Walker is to transform $\mathbf{x}$ to $(\omega, s)$ where $s_{i}=x_{i}^{2}$ and $\omega_{i}=\frac{x_{i}}{\left\|x_{i}\right\|}$, so $\omega_{i}$ can either be 1 or -1 . They then study the marginal and conditional distributions of $\omega$ and $s$.

The $\mathrm{FB}_{8}$ distribution is

$$
p_{F B}(\hat{\mathbf{x}} ; \mathbf{A})=N(\mathbf{b}, \mathbf{A}) \exp \left(\mathbf{b}^{T} \hat{\mathbf{x}}+\hat{\mathbf{x}}^{T} \mathbf{A} \hat{\mathbf{x}}\right)
$$

where $N(\mathbf{b}, \mathbf{A})$ is the normalizing constant, $\mathbf{x} \in R^{3}$ and $\mathbf{x}^{T} \mathbf{x}=1$. We can diagonalize the Bingham matrix $\mathbf{A}$ by a suitable orthogonal transform so we may assume $\mathbf{A}$ is diagonal without any loss of generality i.e. $\mathbf{A}=\operatorname{diag}\left(\lambda_{0}, \lambda_{1}, \lambda_{2}\right)$. Using the parameters $a_{i}=\lambda_{i}-\lambda_{0}$ for $i=1,2$ and $s=1-s_{0}=s_{1}+s_{2}$. We can write the density as

$$
\begin{aligned}
P_{F B}(\omega, s) \propto & \exp \left[\sum_{i=0}^{2}\left(a_{i} s_{i}+b_{i} \omega_{i} \sqrt{s_{i}}\right)\right] \\
& \times \exp \left[b_{0} \omega_{0} \sqrt{1-s}\right] \\
& \times \prod_{i=0}^{2} \frac{1}{\sqrt{s_{i}}} \frac{1}{\sqrt{1-s}} \mathbf{1}(s \leq 1)
\end{aligned}
$$

where, $\mathbf{1}(s \leq 1)$ is the indicator variable and $c$ is a constant. Kume and Walker then introduced three latent variables $(u, v, w)$ and construct a joint density of

$$
\begin{aligned}
f(\omega, s, u, v, w) \propto \mathbf{1} & {\left[u<\exp \left(\sum_{i=1}^{2}\left(a_{i} s_{i}+b_{i} \omega_{i} \sqrt{s_{i}}\right)\right)\right] } \\
& \times \mathbf{1}\left[v<\exp \left(b_{0} \omega_{0} \sqrt{1-s}\right)\right] \\
& \times \mathbf{1}\left[w<\frac{1}{\sqrt{1-s}}\right] \times \prod_{i=1}^{2} \frac{1}{\sqrt{s_{i}}} \mathbf{1}(s \leq 1)
\end{aligned}
$$

Finally, Gibbs sampling may be used to draw samples using the conditional distributions of all of these variables. The values of $s$ and $\omega$ conform to the original Fisher-Bingham distribution and can be converted back into samples of 
$\hat{\mathbf{x}}$. In the interests of space we omit the details of the sampling process which can be found in [9].

We use this slice sampling process to sample a set of surface normals from the $\mathrm{FB}_{8}$ distribution produced by Haines and Wilson shape-from-shading. Typically we sample of the order of 100 normals, which we use in the next section to compute a distribution on the tangent plane. We analyze the number of samples required in the experimental section.

\section{Combining the Normals}

The shape model and the SfS process both provide a distribution for the surface normals at each point in the image. We now explain how we combine these distributions. Quantities from the shape model will be denoted with the subscript 1 and those from the SfS with subscript 2 . These calculations are applied at each pixel.

\subsection{Acquiring the Mean and Covariance Matrices}

Finding the appropriate mean and covariance from Smith's model is straightforward. The mean surface normal is already defined as $\mu_{1}$. We use this direction to define the tangent plane on which we combine the models, because the normals from SfS could potentially be outliers.

The covariance matrix $\mathbf{L}$ from Smith's model describes the covariance on the union of tangent planes at all points. To extract the covariance matrix at a single point, we need only pull out the appropriate components from $\mathbf{L}$ to find the 2by- 2 covariance. We represent this by a precision matrix $\mathbf{P}_{1}$ for convenience:

$$
\begin{gathered}
\mathbf{C}_{1}=\left[\begin{array}{ll}
\mathbf{L}_{x y \alpha, x y \alpha} & \mathbf{L}_{x y \alpha, x y \beta} \\
\mathbf{L}_{x y \alpha, x y \beta} & \mathbf{L}_{x y \beta, x y \beta}
\end{array}\right] \\
\mathbf{P}_{1}=\mathbf{C}_{1}^{-1}
\end{gathered}
$$

where $x y \alpha$ refers to the $\alpha$ component of the tangent-plane normals at pixel $(x, y)$.

For Haines and Wilson SfS, we have $k$ samples from the $\mathrm{FB}_{8}$ distribution for each pixel $\left\{\mathbf{n}_{1}, \mathbf{n}_{2}, \ldots, \mathbf{n}_{k}\right\}$ as described in section 3 . We begin by computing the the extrinsic mean of the surface normal samples using, $\hat{\mathbf{p}}_{\mathbf{0}}=\frac{\mathbf{1}}{\mathbf{K}} \sum_{\mathbf{k = 1}}^{\mathbf{K}} \mathbf{n}_{\mathbf{k}}$. We then use this extrinsic mean to calculate the spherical median using the iterative process below:

$$
\hat{\mathbf{p}}_{j+1}=\operatorname{Exp}_{\hat{\mathbf{p}}_{j}}\left(\frac{1}{K} \sum_{i=1}^{K} \log _{\hat{\mathbf{p}}_{\mathbf{j}}}\left(\mathbf{n}_{\mathbf{i}}\right)\right)
$$

We use 5-10 iterations to calculate the spherical median. Once the spherical median is found we use this surface normal $\mu_{2}=\hat{\mathbf{p}}_{\text {final }}$ as the base point to convert the $k$ samples $\left[\mathbf{n}_{1}, \mathbf{n}_{2}, \ldots, \mathbf{n}_{k}\right]^{T}$ from last iteration to the vectors $\left[\mathbf{v}_{1}, \mathbf{v}_{2}, \ldots, \mathbf{v}_{k}\right]^{T}$ on the tangent plane using the log-map: $\mathbf{v}_{k}=\log _{\mu_{2}}\left(\mathbf{n}_{k}\right)$. From these samples, we compute a tangent-plane covariance $\mathbf{C}_{2}$ and corresponding precision $\mathbf{P}_{2}=\mathbf{C}_{2}^{-1}$ 


\subsection{Combining Gaussian Distributions}

We now have two Gaussian distributions on two different tangent planes corresponding to the SfS model and the shape model. We combine these on a single tangent plane defined by the shape model surface normal as the SfS normal may in some cases be an outlier. The mean of the shape model distribution is of course at the origin on the tangent plane, but we must project the SfS mean onto this tangent plane using the log-map i.e. $\mathbf{v}_{\mu 2}=\log _{\mu_{1}} \mu_{2}$. We may then combine the distributions using the normal rules for combining Gaussians:

$$
\begin{array}{r}
\mathbf{P}^{*}=\mathbf{P}_{1}+\mathbf{P}_{2} \\
\mathbf{v}^{*}=\left(\mathbf{P}^{*}\right)^{-1}\left(\mathbf{P}_{2} \mathbf{v}_{\mu 2}\right)
\end{array}
$$

The normal on the tangent plane is then converted back on the unit-sphere with exponential mapping i.e. $\mu^{*}=\operatorname{Exp}_{\mu_{1}} \mathbf{v}^{*}$. $\mathbf{v}^{*}$ is then our maximum-likelihood estimate of the surface normal.

\subsection{Dealing with Outliers}

Outliers arise in the regions where surface normals resulting from Smith's model do not comply with the surface normals generated through the sampling of $\mathrm{FB}_{8}$ distribution. This is due to the fact that we are so far ignoring the effects of albedo i.e. the irradiance equation assumes unit albedo which does not hold in the eyes, areas of facial hair or shadow regions. To detect these outliers we use Fisher criterion. The Fisher criterion is ideal for our algorithm due to the fact that we have separate class distributions to represent surface normals from shape model and FB sampling.

The Fisher criterion is a linear pattern classifier that evaluates between-class variance relative to the within-class variance 13 . The idea of Fisher criterion lies in finding such a vector $\mathbf{d}$ that the patterns belonging to opposite classes would be optimally separated after projecting them onto $\mathbf{d}$ [14]. The Fisher criterion for two classes can be expressed as

$$
F(\mathbf{d})=\frac{\mathbf{d}^{T} \mathbf{S d}}{\mathbf{d}^{T} \mathbf{\Sigma} \mathbf{d}}
$$

where, $\mathbf{S}$ is between class scatter matrix given by $\mathbf{S}=\Delta \Delta^{T}$ and $\Delta=\mu_{1}-\mu_{2}$. $\mu_{1}, \mu_{2}$ are the mean vectors and $\Sigma_{1}, \Sigma_{2}$ are the covariance matrices of classes 1 and 2. $\Sigma=p_{1} \Sigma_{1}+p_{2} \Sigma_{2}$ where $p_{1}$ and $p_{2}$ are the a priori probabilities of the two classes which are taken as 0.5 while giving equal weightage to both classes. The optimal value of Fisher discriminant $\mathbf{d}_{\text {opt }}$ maximises the value of $F$. Here we are interested in the optimal value of $F\left(\mathbf{d}_{o p t}\right)$ as it gives a good measure of the separation of the two classes. We use the $F\left(\mathbf{d}_{\text {opt }}\right)$ as a threshold to deal with the outliers; the outliers exist at the pixels where the two surface normals from FB sampling and Smith's model do not register with each other. The distributions will therefore be well separated and give a large value of the Fisher criterion. As we already have the necessary class means and covariances to hand, it is a 
straightforward task to compute the criterion. We decide a threshold value for the criterion and then replace that particular pixel's surface normal $\mu^{*}$ with Smith's surface normal $\mu_{1}$ at that specific location if the value of $F\left(\mathbf{d}_{o p t}\right)$ is higher than decided threshold.

\subsection{Surface Reconstruction}

After the surface normals have been combined using product normal distribution we perform smoothing on these normals. Smoothing is required as it finds the normal's best fit to the statistical model. The normals are first converted on the tangent plane using the Smith's statistical normals as the base points using Log map i.e.

$$
u(x, y)=\log _{\mu_{1}(x, y)}\left(\mu^{*}(x, y)\right)
$$

All the image points $[u(1,1), u(1,2), \ldots]$ are then stacked together to give a $N \times 2$ vector $\mathbf{U}_{c}$ of combined normals on the tangent plane. The best fit vector on the tangent plane is then found using 15$]$,

$$
\mathbf{U}_{r}=\mathbf{P} \mathbf{P}^{T} \mathbf{U}_{c}
$$

where $\mathbf{P}$ is the matrix of principal directions from Smith's statistical model. $\mathbf{U}_{c}$ is the vector comprised of combined surface normals on the tangent plane and $\mathbf{U}_{r}$ is the vector of recovered smoothed surface normals. The surface normals on the tangent plane are then brought back to the unit sphere through exponential mapping using Smith's statistical needle map as base points. We will show in experiments that smoothing helps in reducing the RMS error.

Finally we use Smith's height model to recover surface height from surface normals, as described in section 2.3 .

\section{$5 \quad$ Experimental Results}

We have tested our algorithm on the same data set that has been used to construct Smith's statistical models [4]. Iterative Closest Point method [12] was used to compute the RMS distance between ground truth normals and recovered normals and between ground truth surface height and recovered surface height.

\subsection{Effects of Number of Samples}

The only time consuming component in our technique is the slice sampling of the $\mathrm{FB}_{8}$ distributions. For each pixel first 200 samples are used for burn in period then extrinsic mean is calculated with next 100 samples. We use $5-10$ iterations to calculate intrinsic mean; each iteration in turn may use 25, 100, 250 or 500 samples. Increasing the number of samples increases the global consistency of sampled $\mathrm{FB}_{8}$ surface normals resulting from SfS at the expense of time. Figure 1 shows results for one of the test images that has been used as input to the SfS 
algorithm; the outputted $\mathrm{FB}_{8}$ distributions from SfS were sampled using Kume \& Walker [9]. In figure 1 row 1 we have shown the resulting sampled illuminated surface normals when 25,100, 250 and 500 samples were used per iteration for computing spherical median. Row 2 of figure 1 shows the combined normals when SfS sampled normals are combined with statistical model of needle maps. No fisher criterion or smoothing yet have been performed.

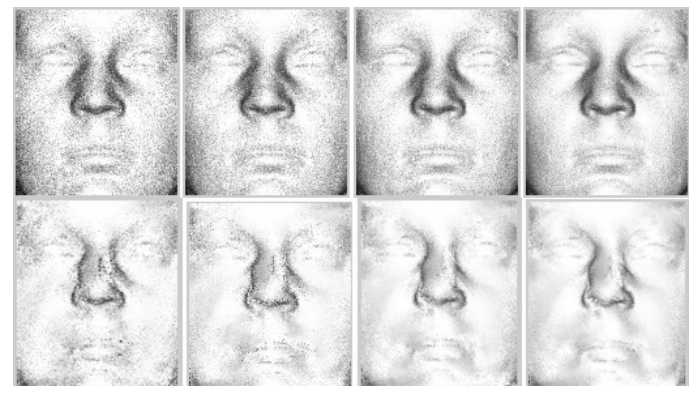

Fig. 1. Illuminated FB Sampled (Row1) and Combined Normals (Row2)

\subsection{Surface Normals and Surface Height Errors}

Figure 2 on the left shows the ground truth normals (Row1) for 5 subjects from our test data, the Shape-from-Shading FB sampled normals (Row2) and resultant Combined normals (Row3). In right half of figure 2 we have shown the illuminated version of these normals when they are illuminated with a frontoparallel unit light source $[0,0,1]$. For subjects shown in figure $2 ; 500$ samples per iteration have been used for computing the spherical median.

We have computed error distances for 200 synthetic images for surface normals and surface heights using Iterative Closest Point algorithm. Figure 3 on the left shows the Root Mean Squared error computed between Groundtruth surface normals and SfS FB sampled normals ( $\mathrm{E}_{\mathrm{FBSampled}}$ shown in red); and between Groundtruth normals and resultant Combined normals $\left(\mathrm{E}_{\mathrm{Combined}}\right.$ shown in green). In the right halft the RMS distance profile computed for surface heights is shown. The number of subjects have been adjusted according to the ascending $\mathrm{E}_{\mathrm{FBSampled}}$ error. Only 25 samples per iteration have been used for spherical median computations for the purpose of computing these errors.

From the error profiles it is apparent that the Root Mean Squared error has reduced significantly when combined surface normals have been used instead of using the surface normals computed through the SfS model alone. So the surface heights constructed from these normals have shown improvements as well as apparent from figure 3 . 


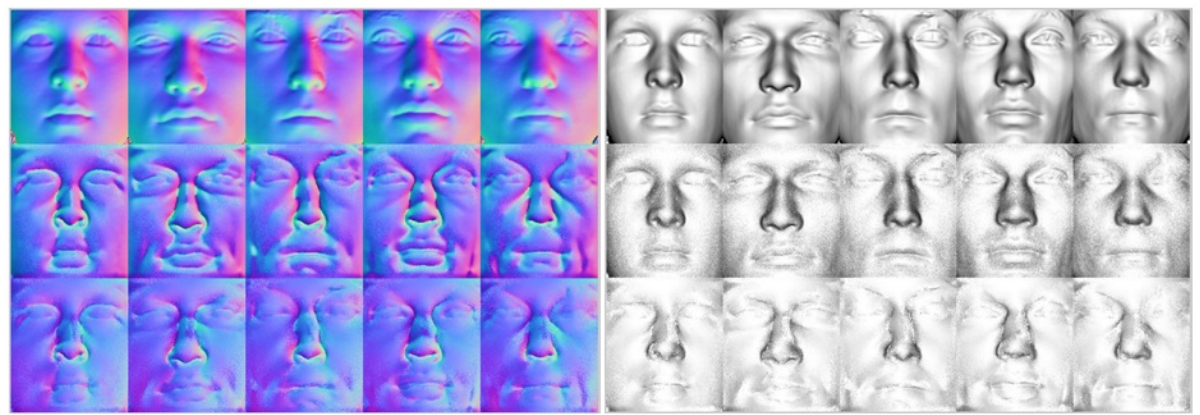

Fig. 2. Groundtruth Surface Normals (Row1), SfS FB Sampled Normals (Row2) and Combined Normals (Row3)
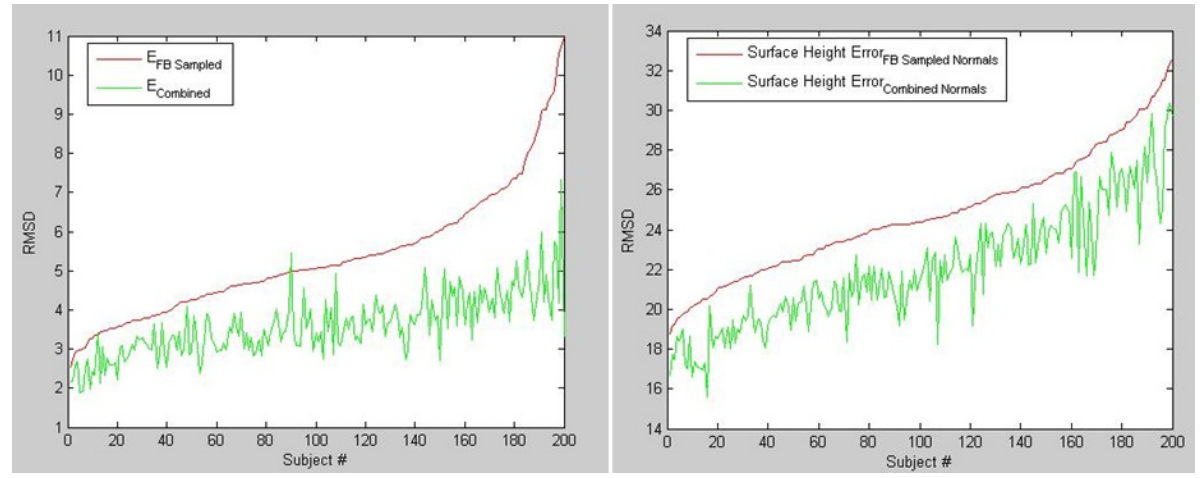

Fig. 3. Profiles of Surface Normal (Left) and Surface Hieght (Right) Errors

\section{Conclusions}

We have presented a probabilistic framework in which Fisher-Bingham 8parameter distributions arising from a probabilistic Shape-from-Shading method are sampled using a slice sampling algorithm; surface normals are calculated from these samples using the machinery of spherical median and log/exp mapping. These normals are then combined with normals resulting from a statistical facial shape model using individual Gaussian distributions on the tangent plane. Fisher criterion and smoothing are used to deal with outliers. From our experiments we have shown that the error distances reduce when combined normals are used; even when less samples from FB sampling were used to compute spherical medians. In our future work we will try to fit Fisher-Bingham distributions to the statistical normals instead of fitting Gaussian distribution to the sampled SfS normals on the tangent plane and then combine the resulting Fisher-Bingham distributions. Since, Fisher-Bingham distribution models directional data more precisely than Gaussian distribution; we hope this will give more reliable combined normals. 


\section{References}

1. Haines, T.S.F., Wilson, R.C.: Belief propagation with directional statistics for solving the shape-from-shading problem. In: Forsyth, D., Torr, P., Zisserman, A. (eds.) ECCV 2008, Part III. LNCS, vol. 5304, pp. 780-791. Springer, Heidelberg (2008)

2. Lee, K.M., Kuo, C.J.: Shape from shading with perspective projection. CVGIP: Image Understanding 59(2), 202-212 (1994)

3. Worthington, P.L., Hancock, E.R.: New constraints on data-closeness and needle map consistency for shape-from-shading. IEEE Trans. Pattern Anal. Intell. 21(12), 1250-1267 (1999)

4. Smith, W.A.P.: Statistical Methods For Facial Shape-from-Shading and Recognition. PhD thesis, University of York (2007)

5. USF HumanID 3D Face Database, Courtesy of Sundeep. Sarkar, University of South Florida, Tampa, FL

6. Blanz, V., Vetter, T.: Face Recognition based on fitting a 3D morphabale model. IEEE Trans. Pattern Anal. Intell. 25(9), 1063-1074 (2003)

7. Fletcher, P.T., Joshi, S., Lu, C., Pizer, S.M.: Principal geodesic analysis for the study of nonlinear statistics of shape. IEEE Trans. Med. Imaging. 23(8), 995-1005 (2004)

8. Pennec, X.: Probabilities and statistics on Riemannian manifolds: basic tools for geometric measurements. In: Proc. IEEE Workshop on Nonlinear Signal and Image Processing (1999)

9. Kume, A., Walker, S.G.: On the Fisher-Bingham distribution. Stat. and Comput. 19, 167-172 (2009)

10. Mardia, K.V., Jupp, P.E.: Directional Statistics. John Wiley and Sons Ltd., Chichester (2000)

11. Felzenszwalb, P.F., Huttenlocher, D.P.: Efficient belief propagation for early vision. In: Computer Vision and Pattern Recognition, vol. 1, pp. 261-268 (2004)

12. Phillips, J.M., Liu, R., Tomasi, C.: Outlier Robust ICP for Minimizing Fractional RMSD. In: 6th International Conference on 3-D Digital Imaging and Modeling, pp. 427-434 (2007)

13. Luebke, K., Weihs, C.: Improving Feature Extraction by Replacing the Fisher Criterion by an Upper Error Bound. Pattern Recognition 38(2005), 2220-2223 (2005)

14. Maciej, S., Witold, M.: Versatile Pattern Recognition System Based on Fisher Criterion. In: Proceedings of the KOSYR, pp. 343-348 (2003)

15. William, A.P.: Smith and Edwin R. Hancock. Facial Shape-from-shading and Recognition Using Principal Geodesic Analysis and Robust Statistics. International Journal of Computer Vision 76(1), 71-91 (2008)

16. Prados, E., Faugeras, O.: Unifying approaches and removing unrealistic assumptions in shape from shading: Mathematics can help. In: Pajdla, T., Matas, J(G.) (eds.) ECCV 2004. LNCS, vol. 3024, pp. 141-154. Springer, Heidelberg (2004)

17. Zhao, W.Y., Chellappa, R.: Symmetric shape-from-shading using self-ratio image. International Journal of Computer Vision 45, 55-75 (2001)

18. Samaras, D., Metaxas, D.: Illumination constraints in deformable models for shape and light direction estimation. IEEE Transactions on Pattern Analysis and Machine Intelligence 25(2), 247-264 (2003) 